\title{
Periodic eigendecomposition and its application to Kuramoto-Sivashinsky system
}

\author{
Xiong Ding ${ }^{\dagger}$ and Predrag Cvitanović ${ }^{\dagger}$
}

\begin{abstract}
Periodic eigendecomposition, to be formulated in this paper, is a numerical method to compute Floquet spectrum and Floquet vectors along periodic orbits in a dynamical system. It is rooted in numerical algorithms advances in computation of 'covariant vectors'* of the linearized flow along an ergodic trajectory in a chaotic system. Recent research on covariant vectors strongly strongly suggests that the physical dimension of inertial manifold of a dissipative PDE can be characterized by a finite number of 'entangled modes', dynamically isolated from the residual set of transient degrees of freedom. We anticipate that Floquet vectors display similar properties as covariant vectors. In this paper we incorporate periodic Schur decomposition to the computation of dynamical Floquet vectors, compare it with other methods, and show that the method can yield the full Floquet spectrum of a periodic orbit at every point along the orbit to high accuracy. Its power, and in particular its ability to resolve eigenvalues whose magnitude differs by hundreds of orders magnitude, is demonstrated by applying the algorithm to computation of the full linear stability spectrum of several periodic solutions in one dimensional Kuramoto-Sivashinsky flow.
\end{abstract}

Key words. periodic eigendecomposition, periodic Schur decomposition, periodic Sylvester equation, covariant vectors, Floquet vectors, Kuramoto-Sivashinsky, linear stability, continuous symmetry

AMS subject classifications. 15A18, 35B10, 37L20, 37M25, 65F15, 65H10, 65P20, 65P40, 76F 20

1. Introduction. In dissipative chaotic dynamical systems, the decomposition of the tangent space of invariant subsets into stable, unstable and center subspaces is important for analyzing the geometrical structure of the solution field [18]. For equilibrium points, the task is quite simple, which is reduced to the eigen-problem of a single stability matrix, but the scenario is much more difficult for complex structures, such as periodic orbits and invariant tours, since the expanding/contracting rate in high dimensional systems usually span a large order of magnitude. Actually, in literature, two different algorithms are capable of resolving this problem partially originated from different settings. The first candidate is covariant vector algorithm [14, 24, 39]. It is designed to stratify the Oseledets subspaces [32] corresponding to the hierarchy of Lyapunov exponents along a long non-wandering orbit on the attractor. Covariant vectors attract a lot of attention in the past few years. They turn out to be a useful tool for physicists to investigate the dynamical properties of the system, such as hyperbolicity degree $[4,19,23]$ and the geometry of inertial manifold [34, 40, 42]. For our interest in periodic orbits, it produces Floquet spectrum and Floquet vectors. The second candidate is called periodic Schur decomposition(PSD) [3], and was brought up to compute the eigenvalues of the product of a sequence of matrices without forming the product explicitly. This is suitable for solving the eigenvalue problem in tangent space because the fundamental matrix in tangent space can be formed as a product of its shorter-time pieces. However, in its original form,

\footnotetext{
${ }^{\dagger}$ Center for Nonlinear Science, School of Physics, Georgia Institute of Technology, Atlanta, GA 30332 (xding@gatech.edu, predrag.cvitanovic@physics.gatech.edu).

*In literature, it is termed "covariant Lyapunov vectors", but we prefer to use covariant vectors in this paper.
} 
PSD are only capable of computing eigenvalues but not eigenvectors. Also PSD seems not well known to the physics community.

In this paper, we unify these two methods for computing Floquet spectrum and Floquet vectors along periodic orbits or invariant tori, and name it after periodic eigendecomposition. Special attention is exerted to complex conjugate Floquet vectors. There are two stages in the process of this algorithm, each of which can be accomplished by two different methods, so we study performance of four different algorithms in all. Also it turns out that the covariant vectors algorithm reduces to one of them when applied to periodic orbits.

The paper is organized as follows. Sect. 2 describes briefly the nonlinear dynamics motivation for undertaking this project, and reviews two existing algorithms related to our work. Readers interested only in the algorithms itself can skip this part. We describe the computational problem in sect. 3 . In sect. 4 we deal with the first stage of periodic eigendecomposition, and then show that both the periodic QR algorithm and simultaneous iteration are capable of achieving periodic Schur decomposition. Sect. 5 introduces power iteration and reordering as two practical methods to obtain all eigenvectors. In sect. 6 we compare the computational effort required by different methods, and sect. 7 applies periodic eigendecomposition to Kuramoto-Sivashinsky equation, an example which illustrates method's effectiveness.

2. Dynamics background and existing algorithms. The study of dynamical systems is trying to understand the statistical properties of the system and the geometrical structure of the global attractor. As we will see, periodic orbits plays an important role in answering both questions. For dissipative systems, orbits typically land onto an invariant subset, called global attractor, after a transient period, and if the system is chaotic, the attractor is a strange attractor which contains a dense set of periodic orbits. The chaotic deterministic flow on strange attractor can be visualized as a walk chaperoned by a hierarchy of unstable invariant solutions (equilibria, periodic orbits) embedded in the attractor. An ergodic trajectory shadows one such invariant solution for a while, is expelled along its unstable manifold, settles into the neighborhood of another invariant solution for a while, and continues in this way forever. Together, the infinite set of these unstable invariant solutions forms the skeleton of a chaotic attractor, and in fact spatiotemporal averages, such as deterministic diffusion coefficients, energy dissipation rate, Lyapunov exponents, etc. can be accurately calculated as a sum over periodic orbits weighted by products of their unstable Floquet multipliers $[5,7]$. This is one reason we study the algorithm of computing Floquet spectrum in this paper.

On the other hand, strange attractor is usually a fractal subset with unsmooth surface, and is not handy to analyze. So this motivates the formulation of concept of inertial manifold [35], which contains the global attractor but is integer-dimensional and exponentially attractive. The existence of inertial manifold has been proved for many dissipative dynamical systems [35], but the mathematical proof shed little knowledge about the dimension of inertial manifold. Although, upper bounds are persistently improved for some systems, such as Kuramoto-Sivashinsky equation [20,31], they are far from being tight and give limited hint for suitable mode truncation in numerical simulations. Recently, however, there is strong numerical evidence [34, 42] that the long-time chaotic (turbulent) dynamics of at least two spatially extended systems, Kuramoto-Sivashinsky and complex Landau-Ginzburg, is confined to an inertial manifold that is everywhere locally spanned by a finite number of 'entangled' modes, dynamically isolated 
from the residual set of isolated, transient degrees of freedom. Covariant vectors exhibit an approximate orthogonality between the 'entangled' modes and the rest, the 'isolated' modes. These results suggest that for a faithful numerical integration of dissipative PDEs, a finite number of entangled modes should suffice, and that increasing the dimensionality beyond that merely increases the number of isolated modes, with no effect on the long-time dynamics. This work has been made possible by advances in algorithms for computation of large numbers of 'covariant vectors' [14, 15, 24, 30, 33, 39]. While these studies offer strong evidence for finite dimensionality of inertial manifolds of dissipative flows, they are based on numerical simulations of long ergodic trajectories and they yield no intuition about the geometry of the attractor. That is attained by studying the hierarchies of unstable periodic orbits, invariant solutions which, together with their Floquet vectors, provide an effective description of both the local hyperbolicity and the global geometry of an attractor embedded in a high-dimensional state space. Motivated by the above studies of covariant vectors, we formulate in this paper a periodic eigendecomposition algorithm suited to accurate computation of Floquet vectors of unstable periodic orbits.

2.1. Linear stability. Now, we turn to the definition of Floquet exponents and Floquet vectors. Let the flow of a autonomous continuous system be described by $\dot{x}=v(x), x \in \mathbb{R}^{n}$ and the corresponding time-forward trajectory starting from $x_{0}$ is $x(t)=f^{t}\left(x_{0}\right)$. In the linear approximation, the deformation of an infinitesimal neighborhood of $x(t)$ (dynamics in tangent space) is governed by the Jacobian matrix (fundamental matrix) $\delta x\left(x_{0}, t\right)=J^{t}\left(x_{0}\right) \delta x\left(x_{0}, 0\right)$, where $J^{t}\left(x_{0}\right)=J^{t-t_{0}}\left(x_{0}, t_{0}\right)=\partial f^{t}\left(x_{0}\right) / \partial x_{0}$. Jacobian matrix satisfies the semi-group multiplicative property (chain rule) along an orbit,

$$
J^{t-t_{0}}\left(x\left(t_{0}\right), t_{0}\right)=J^{t-t_{1}}\left(x\left(t_{1}\right), t_{1}\right) J^{t_{1}-t_{0}}\left(x\left(t_{0}\right), t_{0}\right) .
$$

For a periodic point $x$ on orbit $p$ of period $T_{p}, J_{p}=J^{T_{p}}(x)$ is called the Floquet matrix (monodromy matrix) and its eigenvalues the Floquet multipliers $\Lambda_{j}$. A Floquet multiplier is a dimensionless ratio of the final/initial perturbation along the $j_{t h}$ eigen-direction. It is an intrinsic, local property of a smooth flow, invariant under all smooth coordinate transformations. The associated Floquet vectors $\mathbf{e}_{j}(x), J_{p} \mathbf{e}_{j}=\Lambda_{j} \mathbf{e}_{j}$, define the invariant directions of the tangent space at the periodic point $x=x(t) \in p$. Evolving small initial perturbation aligned with a Floquet direction will generate the corresponding unstable manifold along the periodic orbit. Floquet multipliers are either real, $\Lambda_{j}=\sigma_{j}\left|\Lambda_{j}\right|, \sigma_{j} \in\{1,-1\}$, or form complex pairs, $\left\{\Lambda_{j}, \Lambda_{j+1}\right\}=\left\{\left|\Lambda_{j}\right| \exp \left(i \theta_{j}\right),\left|\Lambda_{j}\right| \exp \left(-i \theta_{j}\right)\right\}, 0<\theta_{j}<\pi$. The real parts of Floquet exponents $\mu_{j}=\left(\ln \left|\Lambda_{j}\right|\right) / T_{p}$ describe the mean contraction or expansion rates per one period of the orbit. The Jacobian matrix is naively obtained numerically by integrating the stability matrix

$$
\frac{d J^{t}}{d t}=A(x) J^{t}, \quad \text { with } \quad A(x)=\frac{\partial v(x)}{\partial x}
$$

along the orbit. However, it is almost certain that this process will overflow or underflow at exponential rate as the system evolves or the resulting Jacobian is highly ill-conditioned. Thus, accurate calculation of expansion rate is not trivial for nonlinear systems, especially for those that evolve in a high dimensional space. In such cases, the expansion/contraction rate 
can easily range over many orders of magnitude, which raises a challenge to formulating an effective algorithm to tackle this problem. However, the semi-group property (2.1) enables us to factorize the Jacobian matrix into a product of short-time matrices with matrix elements of comparable magnitudes. So the problem is reduced to calculating the eigenvalues of the product of a sequence of matrices.

2.2. covariant vectors. multiplicative ergodic theorem [29, 32] says that the forward and backward Oseledets matrices

$$
\Lambda^{ \pm}(x):=\lim _{t \rightarrow \pm \infty}\left[J^{t}(x)^{\top} J^{t}(x)\right]^{1 / 2 t}
$$

both exist for an invertible dynamical system equipped with an invariant measure. The eigenvalues are $e^{\lambda_{1}^{ \pm}(x)}<\cdots<e^{\lambda_{s}^{ \pm}(x)}$, where $\lambda_{i}^{ \pm}(x)$ are the Lyapunov exponents (characteristic exponents) and $s$ is the total number of distinct exponents $(s \leq n)$. For an ergodic system, Lyapunov exponents are the same almost everywhere, and $\lambda_{i}^{+}(x)=-\lambda_{s-i+1}^{-}(x)=\lambda_{i}$. The corresponding eigenspaces $U_{1}^{ \pm}(x), \cdots, U_{s}^{ \pm}(x)$ can be used to construct the forward and backward invariant subspaces: $V_{i}^{+}(x)=U_{1}^{+}(x)+\cdots+U_{i}^{+}(x), V_{i}^{-}(x)=U_{s}^{-}(x)+\cdots+U_{s-i+1}^{-}$. So the intersections $W_{i}(x)=V_{i}^{+}(x) \cap V_{i}^{-}(x)$ are dynamically forward and backward invariant: $J^{ \pm t}(x) W_{i}(x) \rightarrow W_{i}\left(f^{ \pm t}(x)\right), i=1,2, \cdots, s$. The expansion rate in invariant subspace $W_{i}(x)$ is given by the corresponding Lyapunov exponents,

$$
\lim _{t \rightarrow \pm \infty} \frac{1}{|t|} \ln \left\|J^{t}(x) u\right\|=\lim _{t \rightarrow \pm \infty} \frac{1}{|t|} \ln \left\|\left[J^{t}(x)^{\top} J^{t}(x)\right]^{1 / 2} u\right\|= \pm \lambda_{i}, \quad u \in W_{i}(x)
$$

If a Lyapunov exponent has degeneracy one, the corresponding subspace $W_{i}(x)$ reduces to a vector, called covariant vector. For periodic orbits, these $\lambda_{i}$ (evaluated numerically as $t \rightarrow \infty$ limits of many repeats of the prime period $T$ ) coincide with the real part of Floquet exponents (computed in one period of the orbit). Subspace $W_{i}(x)$ coincides with a Floquet vector, or, if there is degeneracy, a subspace spanned by Floquet vectors.

The reorthonormalization procedure formulated by Benettin etc. [2] is the standard way to calculate the full spectrum of Lyapunov exponents, and it is shown [10] that the orthogonal vectors produced at the end of calculation converges to $U_{i}^{-}$, eigenvectors of $\Lambda^{-}(x)$, called the GS vectors (backward Lyapunov vectors). Based on this technique, Wolf etc. [39] and Ginelli etc. [14] invented independent methods to recover covariant vectors from GS vectors. Here, we should emphasize that GS vectors are not invariant. Except the leading one, all of them are dependent on the specific inner product imposed by the dynamics. Also the local expansion rate of covariant vectors are not identical to the local expansion rate of GS vectors. Specifically for periodic orbits, Floquet vectors depend on no norm, and map forward and backward as $\mathbf{e}_{j} \rightarrow J \mathbf{e}_{j}$ under time evolution. In contrast, the linearized dynamics does not transport GS vectors into the tangent space computed further downstream. For more detailed comparison, please see [24, 41].

2.3. Covariant vectors algorithm. Here we briefly introduce the method used by Ginelli etc. to extract covariant vectors from GS vectors. The setup is the same as computing Lyapunov exponents. We follow a long ergodic trajectory, and integrate the linearized dynamics in tangent space (2.2) with periodic orthonormalization, shown as the first two stages in figure 1 . Here, $J_{i}$ is the Jacobian matrix corresponding to time interval $\left(t_{i}, t_{i+1}\right)$, and diagonal 


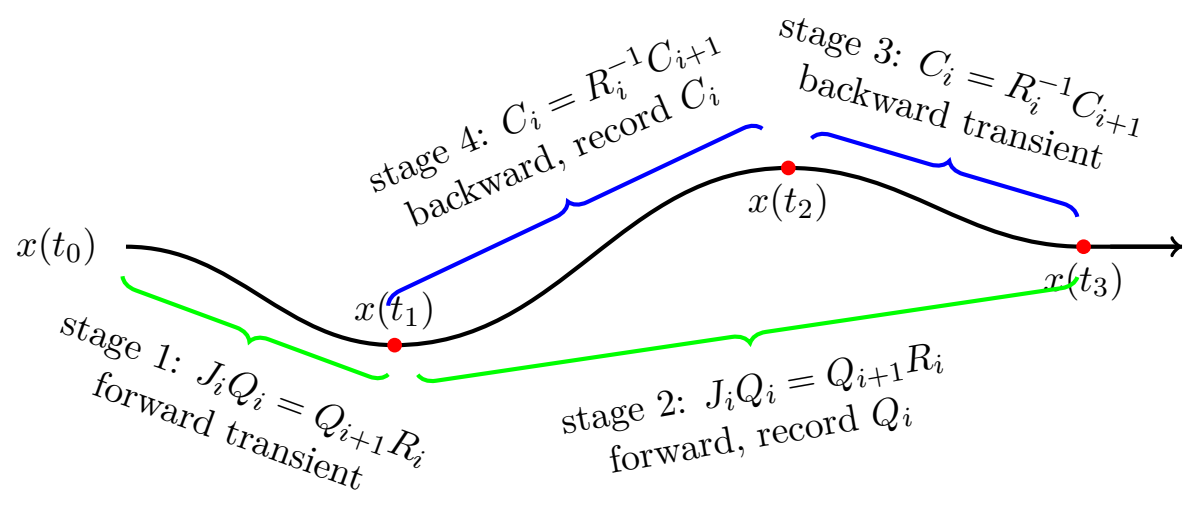

Figure 1. Four stages of Covariant vectors algorithm. The black line is a part of a long ergodic trajectory.

elements of upper-triangular matrices $R_{i}$ store local Lyapunov exponents, long time average of which gives the Lyapunov exponents of this system. We assume $Q_{i}$ converges to the GS vectors after stage 1 , and start to record $R_{i}$ in stage 2 . Since the first $m$ GS vectors span the same subspace as the first $m$ covariant vectors, which means $W_{i}=Q_{i} C_{i}^{\dagger}{ }^{\dagger}$ with $C_{i}$ an upper-triangular matrix, giving the expansion coefficients of covariant vectors in the GS basis. So we have $W_{i}=J_{i-1} Q_{i-1} R_{i}^{-1} C_{i}=J_{i-1} W_{i-1} C_{i-1}^{-1} R_{i}^{-1} C_{i}$. Since $W_{i}$ is invariant in the tangent space, we must have $C_{i-1}^{-1} R_{i}^{-1} C_{i}=I$, which gives the backward dynamics of matrix $C_{i}: C_{i-1}=R_{i}^{-1} C_{i}$. Ginelli etc. cleverly uncover this backward dynamics and show that $C_{i}$ converges after a sufficient number of iterations (stage 3 in figure 1). This process is continued in stage 4 in figure 1 , and $C_{i}$ are recorded in this stage. Finally, we obtain the covariant vectors for trajectory $x\left(t_{1}\right)$ to $x\left(t_{2}\right)$ in figure 1 .

covariant vectors algorithm is invented to stratify the tangent spaces along an ergodic trajectory, so it is hard to observe degeneracy numerically. However, for periodic orbits, it is possible that some Floquet vectors form conjugate complex pairs. When this algorithm is applied to periodic orbits, it is reduced to a combination of simultaneous iteration and pure power iteration; consequently, complex conjugate pairs cannot be told apart. This means that we need to pay attention to the two dimensional rotation when checking the convergence of each stages in figure 1. As is shown in latter sections, a complex conjugate pair of Floquet vectors can be extracted from this converged two dimensional subspace.

2.4. Periodic Schur decomposition algorithm. The implicit QR algorithm (Francis's algorithm) is the standard way of solving eigen problem of a single matrix in many numerical packages, such as the eig () function in Matlab. Bojanczyk etc. [3] extends the idea to compute eigenvalues of the product of a sequence of matrices. Later on, Kurt Lust [26] describes the implementation details and provides the corresponding Fortran code. As stated before, by use of chain rule (2.1), Jacobian matrix can be decomposed into a product of short-time Jacobians with the same dimension, so periodic Schur decomposition is suitable for computing Floquet

\footnotetext{
${ }^{\dagger}$ Here, $W_{i}$ refers to the matrix formed by individual covariant vectors at step $i$ of the algorithm. Do not get confused with the $i t h$ covariant vector
} 


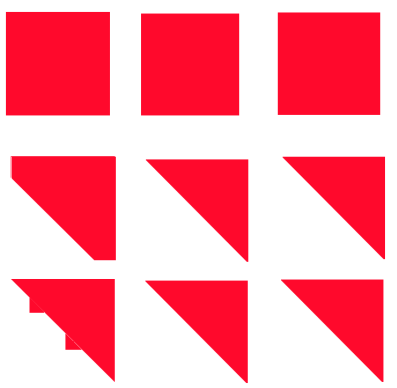

Figure 2. Two stages of periodic Schur decomposition algorithm illustrated by tree matrices.

exponents, and we think it is necessary to introduce this algorithm into physics community.

As illustrated in figure 2, periodic Schur decomposition proceeds in two stages. First, the sequence of matrices are transformed to Hessenberg-Triangular form, one of which has upperHessenberg form while the others are upper-triangular, by a series of Household reflections. The second stage is iteration of periodic QR algorithm, which diminishes the sub-diagonal components of the Hessenberg matrix until it becomes quasi-upper-triangular. The convergence of this second stage is guaranteed by "Implicit Q Theorem" [12,38]. After the second stage, The sequence of matrices are all transformed into upper-triangular form except one of them be quasi-upper triangular - there are some $[2 \times 2]$ blocks on the diagonal corresponding to complex eigenvalues. Then the eigenvalues are the product of their diagonal elements. However, periodic Schur decomposition is not enough for extracting eigenvectors except the leading one. Kurt Lust claims to formulate the corresponding Floquet vector algorithm, but to the best of our knowledge, such algorithm is not present in literature. Fortunately, Granat etc. [17] propose a method to reorder the diagonal elements after periodic Schur decomposition. It provides a elegant way to compute Floquet vectors as we will see in later sections.

3. Description of the problem. After introducing the underlying physical motivation, let us turn to the definition of the problem. According to (2.1), Jacobian matrix can be integrated piece by piece along a state orbit:

$$
J^{t}\left(x_{0}\right)=J^{t_{m}-t_{m-1}}\left(x\left(t_{m-1}\right), t_{m-1}\right) \cdots J^{t_{2}-t_{1}}\left(x\left(t_{1}\right), t_{1}\right) J^{t_{1}-t_{0}}\left(x\left(t_{0}\right), t_{0}\right)
$$

with $t_{0}=0, t_{m}=t$ and $x_{0}$ the initial point. For periodic orbits, $x\left(t_{m}\right)=x_{0}$. The time sequence $t_{i}, i=1,2, \cdots, m-1$ is chosen properly such that the elements of each Jacobian matrix associated with each small time interval has relatively similar order of magnitude. For simplicity, we drop all the parameters above and use a bold letter to denote the product:

$$
\mathbf{J}^{(0)}=J_{m} J_{m-1} \cdots J_{1}, \quad J_{i} \in \mathbb{R}^{n \times n}, i=1,2, \cdots, m .
$$

This product can be diagonalized if and only if the sum of dimensions of eigenspaces of $\mathbf{J}^{(0)}$ is $n$.

$$
\mathbf{J}^{(0)}=E^{(0)} \Sigma\left(E^{(0)}\right)^{-1},
$$

where $\Sigma$ is a diagonal matrix which stores $\mathbf{J}^{(0)}$ 's eigenvalues (Floquet multipliers), $\left\{\Lambda_{1}, \Lambda_{2}, \cdots, \Lambda_{n}\right\}$, and columns of matrix $E^{(0)}$ are the eigenvectors (Floquet vectors) of $\mathbf{J}^{(0)}: E^{(0)}=\left[\mathbf{e}_{1}^{(0)}, \mathbf{e}_{2}^{(0)}, \cdots, \mathbf{e}_{n}^{(0)}\right]$. 
In this paper all vectors are written in the column form, transpose of $v$ is denoted $v^{\top}$, and Euclidean 'dot' product by $\left(v^{\top} u\right)$. The challenge associated with obtaining diagonalized form (3.2) is the fact that often $\mathbf{J}^{(0)}$ should not be written explicitly since the integration process (2.2) may overflow or the resulting matrix is highly ill-conditioned. Floquet multipliers can easily vary over 100's orders of magnitude, depending on the system under study and the period of the orbit; therefore all transformations should be applied to the short time Jacobian matrices $J_{i}$ individually, instead of working with the full-time $\mathbf{J}^{(0)}$. Also, in order to characterize the geometry along a periodic orbit, not only the Floquet vectors at the initial point are required, but also the sets at each point on the orbit. Therefore, we also desire the eigendecomposition of the cyclic rotations of $\mathbf{J}^{(0)}: \mathbf{J}^{(k)}=J_{k} J_{k-1} \cdots J_{1} J_{m} \cdots J_{k+1}$ for $k=1,2, \ldots, m-1$. Eigendecomposition of all $\mathbf{J}^{(k)}$ is called the periodic eigendecomposition of the matrix sequence $J_{m}, J_{m-1}, \cdots, J_{1}$.

The process of implementing eigendecomposition (3.2) proceeds in two stages. First, periodic real Schur form (PRSF) is obtained by a similarity transformation for each $J_{i}$,

$$
J_{i}=Q_{i} R_{i} Q_{i-1}^{\top},
$$

with $Q_{i}$ orthogonal matrix, and $Q_{0}=Q_{m}$. In the case considered here, $R_{m}$ is quasi-upper tri-

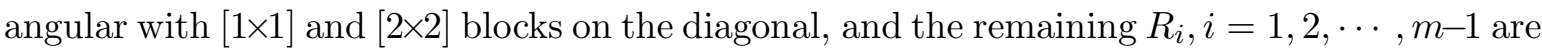
upper triangular. The existence of PRSF, proved in ref. [3], provides the periodic QR algorithm that implements periodic Schur decomposition. Defining $\mathbf{R}^{(k)}=R_{k} R_{k-1} \cdots R_{1} R_{m} \cdots R_{k+1}$, we have

$$
\mathbf{J}^{(k)}=Q_{k} \mathbf{R}^{(k)} Q_{k}^{\top},
$$

with the eigenvectors of matrix $\mathbf{J}^{(k)}$ related to eigenvectors of quasi-upper triangular matrix $\mathbf{R}^{(k)}$ by orthogonal matrix $Q_{k} . \mathbf{J}^{(k)}$ and $\mathbf{R}^{(k)}$ have the same eigenvalues, stored in the $[1 \times 1]$ and [2×2] blocks on the diagonal of $\mathbf{R}^{(k)}$, and their eigenvectors are transformed by $Q_{k}$, so the second stage concerns the eigendecomposition of $\mathbf{R}^{(k)}$. Eigenvector matrix of $\mathbf{R}^{(k)}$ has the same structure as $R_{m}$. We evaluate it by two distinct algorithms. The first one is power iteration, while the second algorithm relies on solving a periodic Sylvester equation [17].

As all $\mathbf{R}^{(k)}$ have the same eigenvalues, and their eigenvectors are related by similarity transformations,

$$
\mathbf{R}^{(k)}=\left(R_{m} \cdots R_{k+1}\right)^{-1} \mathbf{R}^{(0)}\left(R_{m} \cdots R_{k+1}\right),
$$

one may be tempted to calculate the eigenvectors of $\mathbf{R}^{(0)}$, and obtain the eigenvectors of $\mathbf{R}^{(k)}$ by (3.5). The pitfall of this approach is that numerical errors accumulate when multiplying a sequence of upper triangular matrices, especially for large $k$. Therefore, in the second stage of implementing periodic eigendecomposition, iteration is needed for each $\mathbf{R}^{(k)}$ if power iteration method is chosen in this stage. Periodic Sylvester equation bypasses this problem by giving the eigenvectors of all $\mathbf{R}^{(k)}$ simultaneously.

Our work illustrates the connection between different algorithms in the two stages of implementing periodic eigendecomposition, pays attention to the case when eigenvectors appear as complex pairs, and demonstrates that eigenvectors can be obtained directly from periodic Sylvester equation without restoring PRSF. 
4. Stage 1 : periodic real Schur form (PRSF). This is the first stage of implementing periodic eigendecomposition. Eq. (3.4) represents the eigenvalues of matrix $\mathbf{J}^{(k)}$ as real eigenvalues on the diagonal, and complex eigenvalue pairs as $[2 \times 2]$ blocks on the diagonal of $\mathbf{R}^{(k)}$. More specific, if the $i_{t h}$ eigenvalue is real, it is given by the product of all the $i_{t h}$ diagonal elements of matrices $R_{1}, R_{2}, \cdots, R_{m}$. In practice, the logarithms of magnitudes of these numbers are added, in order to overcome numerical overflows. If the $i_{t h}$ and $(i+1)_{t h}$ eigenvalues form a complex conjugate pair, all $[2 \times 2]$ matrices at position $(i, i+1)$ on the diagonal of $R_{1}, R_{2}, \cdots, R_{m}$ are multiplied with normalization at each step, and the two complex eigenvalues of the product are obtained. There is no danger of numerical overflow because all these $[2 \times 2]$ matrices are in the same position and in our applications their elements are of similar order of magnitude. Sec. 2.4 introduce the periodic Schur decomposition to achieve PRSF. Another alternative is the first two stages of covariant vectors in sec. 2.3, which reduces to simultaneous iteration for periodic orbits. Actually, these two methods are equivalent [37], but the computational complexity differs.

Simultaneous iteration. The basic idea of simultaneous iteration is implementing QR decomposition in the process of power iteration. Assume all Floquet multipliers are real, without degeneracy, and order them by their magnitude: $\left|\Lambda_{1}\right|>\left|\Lambda_{2}\right|>\cdots>\left|\Lambda_{n}\right|$, with corresponding normalized Floquet vectors $\mathbf{e}_{1}, \mathbf{e}_{2}, \cdots, \mathbf{e}_{n}$. For simplicity, here we have dropped the upper indices of these vectors. An arbitrary initial vector $\tilde{q}_{1}=\sum_{i=1}^{n} \alpha_{i}^{(1)} \mathbf{e}_{i}$ will converge to the first Floquet vector $\mathbf{e}_{1}$ after normalization under power iteration of $\mathbf{J}^{(0)}$,

$$
\lim _{\ell \rightarrow \infty} \frac{\left(\mathbf{J}^{(0)}\right)^{\ell} \tilde{q}_{1}}{\|\cdot\|} \rightarrow q_{1}=\mathbf{e}_{1}
$$

Here $\|\cdot\|$ denotes the Euclidean norm of the numerator $\left(\|x\|=\sqrt{x^{\top} x}\right)$. Let $\langle a, b, \cdots, c\rangle$ represent the space spanned by vector $a, b, \cdots, c$ in $\mathbb{R}^{n}$. Another arbitrary vector $\tilde{q}_{2}$ is then chosen orthogonal to subspace $\left\langle q_{1}\right\rangle$ by Gram-Schmidt orthonormalization, $\tilde{q}_{2}=\sum_{i=2}^{n} \alpha_{i}^{(2)}\left[\mathbf{e}_{i}-\right.$ $\left.\left(q_{1}^{\top} \mathbf{e}_{i}\right) q_{1}\right]$. Note that the index starts from $i=2$ because $\left\langle q_{1}\right\rangle=\left\langle v_{1}\right\rangle$. The strategy now is to apply power iteration of $\mathbf{J}^{(0)}$ followed by orthonormalization in each iteration.

$$
\begin{aligned}
\mathbf{J}^{(0)} \tilde{q}_{2} & =\sum_{i=2}^{n} \alpha_{i}^{(2)}\left[\Lambda_{i} \mathbf{e}_{i}-\Lambda_{1}\left(q_{1}^{\top} \mathbf{e}_{i}\right) q_{1}\right] \\
& =\sum_{i=2}^{n} \alpha_{i}^{(2)} \Lambda_{i}\left[\mathbf{e}_{i}-\left(q_{1}^{\top} \mathbf{e}_{i}\right) q_{1}\right]+\sum_{i=2}^{n} \alpha_{i}^{(2)}\left(\Lambda_{i}-\Lambda_{1}\right)\left(q_{1}^{\top} \mathbf{e}_{i}\right) q_{1} .
\end{aligned}
$$

The second term in the above expression will disappear after performing Gram-Schmidt orthonormalization to $\left\langle q_{1}\right\rangle$, and the first term will converge to $q_{2}=\mathbf{e}_{2}-\left(q_{1}^{\top} \mathbf{e}_{2}\right) q_{1}$ (not normalized) after a sufficient number of iterations because of the decreasing magnitudes of $\Lambda_{i}$, and we also note that $\left\langle v_{1}, v_{2}\right\rangle=\left\langle q_{1}, q_{2}\right\rangle$. The same argument can be applied to $\tilde{q}_{i}, i=3,4, \cdots, n$ as well. In this way, after a sufficient number of iterations,

$$
\lim _{\ell \rightarrow \infty}\left(\mathbf{J}^{(0)}\right)^{\ell}\left[\tilde{q}_{1}, \tilde{q}_{2}, \cdots, \tilde{q}_{n}\right] \rightarrow\left[q_{1}, q_{2} \cdots, q_{n}\right],
$$

where

$$
q_{1}=\mathbf{e}_{1}, \quad q_{2}=\frac{\mathbf{e}_{2}-\left(\mathbf{e}_{2}^{\top} q_{1}\right) q_{1}}{\|\cdot\|}, \quad \cdots, \quad q_{n}=\frac{\mathbf{e}_{n}-\sum_{i=1}^{n-1}\left(\mathbf{e}_{n}^{\top} q_{i}\right) q_{i}}{\|\cdot\|}
$$


Let matrix $Q_{0}=\left[q_{1}, q_{2}, \cdots, q_{n}\right]$; then we have $\mathbf{J}^{(0)} Q_{0}=Q_{0} \mathbf{R}^{(0)}$ with $\mathbf{R}^{(0)}$ an upper triangular matrix because of $\left\langle q_{1}, q_{2}, \cdots, q_{i}\right\rangle=\left\langle v_{1}, v_{2}, \cdots, v_{i}\right\rangle$, which is just $\mathbf{J}^{(0)}=Q_{0} \mathbf{R}^{(0)} Q_{0}^{\top}$ (the Schur decomposition of $\left.\mathbf{J}^{(0)}\right)$. The diagonal elements of $\mathbf{R}^{(0)}$ are the eigenvalues of $\mathbf{J}^{(0)}$ in decreasing order. Numerically, the process described above can be implemented on an arbitrary initial full rank matrix $\tilde{Q}_{0}$ followed by QR decomposition at each step $J_{s} \tilde{Q}_{s-1}=\tilde{Q}_{s} \tilde{R}_{s}$ with $s=1,2,3, \cdots$ and $J_{s+m}=J_{s}$. For sufficient number of iterations, $\tilde{Q}_{s}$ and $\tilde{R}_{s}$ converge to $Q_{s}$ and $R_{s}(3.3)$ for $s=1,2, \cdots, n$, so we achieve (3.4) the periodic Schur decomposition of $\mathbf{J}^{(k)}$.

We have thus demonstrated that simultaneous iteration converges to PRSF for real nondegenerate eigenvalues. For complex eigenvalue pairs, the algorithm converges in the sense that the subspace spanned by a complex conjugate vector pair converges. So,

$$
\mathbf{J}^{(0)} Q_{0}=Q_{0}^{\prime} \mathbf{R}^{(0)}=Q_{0} D \mathbf{R}^{(0)},
$$

where $D$ is a block-diagonal matrix with diagonal elements \pm 1 (corresponding to real eigenvalues) or $[2 \times 2]$ blocks (corresponding to complex eigenvalue pairs). Absorb $D$ into $R_{m}$, then $R_{m}$ becomes a quasi-upper triangular matrix, and (3.3) still holds.

5. Stage 2 : eigenvector algorithms. Upon achieving PRSF, the eigenvectors of $\mathbf{J}^{(k)}$ are related to eigenvectors of $\mathbf{R}^{(k)}$ by orthogonal matrix $Q_{k}$ from (3.3), and the eigenvector matrix of $\mathbf{R}^{(k)}$ has the same quasi-upper triangular structure as $R_{m}$. In addition, if we follow the simultaneous iteration method or implement periodic Schur decomposition without shift, eigenvalues are ordered by their magnitudes on the diagonal. Power iteration utilizing this property could be easily implemented to generate the eigenvector matrix. This is the basic idea of the first algorithm for generating eigenvectors of $\mathbf{R}^{(k)}$, corresponding to the $3 \mathrm{rd}$ and 4 th stage in covariant vectors algorithm in figure figure 1 . Alternatively, observation that the first eigenvector of $\mathbf{R}^{(k)}$ is trivial if it is real, $v_{1}=(1,0, \cdots, 0)^{\top}$, inspires us to reorder the eigenvalues so that the $j_{t h}$ eigenvalue is in the first diagonal place of $\mathbf{R}^{(k)}$; in this way, the $j_{t h}$ eigenvector is obtained. For both methods, attention should be paid to the complex conjugate eigenvector pairs. In this section, $v_{i}^{(k)}$ denotes the $i_{t h}$ eigenvectors of $\mathbf{R}^{(k)}$, contrast to $\mathbf{e}_{i}^{(k)}$ the eigenvectors of $\mathbf{J}^{(k)}$, and for most cases, the upper indices are dropped if no confusion occurs.

5.1. Iteration method. The prerequisite for iteration method is that all the eigenvalues are ordered in a ascending or descending way by their magnitude on the diagonal of $\mathbf{R}^{(k)}$. Assume that they are in descending order, which is the outcome of simultaneous iteration; therefore the diagonal elements of $\mathbf{R}^{(k)}$ are $\Lambda_{1}, \Lambda_{2}, \cdots, \Lambda_{n}$, with magnitudes from large to small. If the $i_{t h}$ eigenvector of $\mathbf{R}^{(k)}$ is real, then it has form $v_{i}=\left(a_{1}, a_{2}, \cdots, a_{i}, 0, \cdots, 0\right)^{\top}$. An arbitrary vector whose first $i$ elements are nonzero $x=\left(b_{1}, b_{2}, \cdots, b_{i}, 0, \cdots, 0\right)^{\top}$ is a linear combination of the first $i$ eigenvectors: $x=\sum_{j=1}^{i} \alpha_{j} v_{j}$. Use it as the initial condition for the power iteration by $\left(\mathbf{R}^{(k)}\right)^{-1}=R_{k+1}^{-1} \cdots R_{m}^{-1} R_{1}^{-1} R_{2}^{-1} \cdots R_{k}^{-1}$ and after sufficient number of iterations,

$$
\lim _{\ell \rightarrow \infty} \frac{\left(\mathbf{R}^{(k)}\right)^{-\ell} x}{\|\cdot\|}=v_{i}
$$

The property we used here is that $\left(\mathbf{R}^{(k)}\right)^{-1}$ and $\mathbf{R}^{(k)}$ have the same eigenvectors but inverse eigenvalues. 
For a [2×2] block on the diagonal of $\mathbf{R}^{(k)}$, the corresponding conjugate complex eigenvectors form a two dimensional subspace. Any real vector selected from this subspace will rotate under power iteration. In this case, power iteration still converges in the sense that the subspace spanned by the complex conjugate eigenvector pair converges. Suppose the $i_{t h}$ and $(i+1)_{t h}$ eigenvectors of $\mathbf{R}^{(k)}$ form a complex pair. Two arbitrary vectors $x_{1}$ and $x_{2}$ whose first $i+1$ elements are non zero can be written as the linear superposition of the first $i+1$ eigenvectors, $x_{1,2}=\left(\sum_{j=1}^{i-1} \alpha_{j}^{(1,2)} v_{j}\right)+\alpha_{i}^{(1,2)} v_{i}+\left(\alpha_{i}^{(1,2)} v_{i}\right)^{*}$, where $(*)$ denotes the complex conjugate. As for the real case, the first $i-1$ components above will vanish after a sufficient number of iterations. Denote the two vectors at this instance (corresponding to $x_{1,2}$ ) to be $X_{1}$ and $X_{2}$ and form matrix $X=\left[X_{1}, X_{2}\right]$. The subspace spanned by $X_{1,2}$ does not change and $X$ will be rotated after another iteration,

$$
\left(\mathbf{R}^{(k)}\right)^{-1} X=X^{\prime}=X C,
$$

where $C$ is a $[2 \times 2]$ matrix which has two complex conjugate eigenvectors $v_{C}$ and $\left(v_{C}\right)^{*}$. Transformation (5.1) relates the eigenvectors of $\mathbf{R}^{(k)}$ with those of $C:\left[v_{i},\left(v_{i}\right)^{*}\right]=X\left[v_{C},\left(v_{C}\right)^{*}\right]$. In practice, matrix $C$ can be computed by $\mathrm{QR}$ decomposition; let $X=Q_{X} R_{X}$ be the QR decomposition of $X$, then $C=R_{X}^{-1} Q_{X}^{\top} X^{\prime}$. On the other hand, complex eigenvectors are not uniquely determined in the sense that $e^{i \theta} v_{i}$ is also a eigenvector with the same eigenvalue as $v_{i}$ for an arbitrary angle $\theta$, so when comparing results from different eigenvector algorithms, we need a constraint to fix the phase of a complex eigenvector, such as letting the first element be real.

We should note that performance of power iteration depends on the ratios of magnitudes of eigenvalues, so performance is poor for systems with clustered eigenvalues. We assume that proper modifications, such as shifted iteration or inverse iteration, may help improve the performance. Such techniques are beyond the scope of this paper.

5.2. reordering method. Except for the performance problem with clustered eigenvalues, the power iteration has a more severe issue when applied to dynamical systems, that is, it cannot get the eigenvectors of $\mathbf{R}^{(k)}$ for all $k \in 0,1,2, \cdots, m$ at the same time. Although eigenvectors of $\mathbf{R}^{(k)}$ and $\mathbf{R}^{(0)}$ are related by (3.5), it is not advisable, as pointed out above, to evolve the eigenvectors of $\mathbf{R}^{(0)}$ so as to get eigenvectors of $\mathbf{R}^{(k)}$ because of the noise introduced during this process. Therefore, iteration is needed for each $k \in 0,1,2, \cdots, m$.

There exists a direct algorithm to obtain the eigenvectors of every $\mathbf{R}^{(k)}$ at once without iteration. The idea is very simple: the eigenvector corresponding to the first diagonal element of an upper-triangular matrix is $v_{1}=(1,0, \cdots, 0)^{\top}$. By reordering the diagonal elements (or $[2 \times 2]$ blocks) of $\mathbf{R}^{(0)}$, we can find any eigenvector by positioning the corresponding eigenvalue in the first diagonal position. Although in our application only reordering of $[1 \times 1]$ and $[2 \times 2]$ blocks is needed, we recapitulate here the general case of reordering two adjacent blocks of a quasi-upper triangular matrix following Granat [17]. Partition $R_{i}$ as

$$
R_{i}=\left[\begin{array}{c|cc|c}
R_{i}^{00} & * & * & * \\
\hline 0 & R_{i}^{11} & R_{i}^{12} & * \\
0 & 0 & R_{i}^{22} & * \\
\hline 0 & 0 & 0 & R_{i}^{33}
\end{array}\right]
$$


where $R_{i}^{00}, R_{i}^{11}, R_{i}^{22}, R_{i}^{33}$ have size $\left[p_{0} \times p_{0}\right],\left[p_{1} \times p_{1}\right],\left[p_{2} \times p_{2}\right]$ and $\left[p_{3} \times p_{3}\right]$ respectively, and $p_{0}+p_{1}+p_{2}+p_{3}=n$. In order to exchange the middle two blocks $\left(R_{i}^{11}\right.$ and $\left.R_{i}^{22}\right)$, we construct a non-singular periodic matrix sequence: $\hat{S}_{i}, i=0,1,2, \cdots, m$ with $\hat{S}_{0}=\hat{S}_{m}$,

$$
\hat{S}_{i}=\left[\begin{array}{c|c|c}
I_{p_{0}} & 0 & 0 \\
\hline 0 & S_{i} & 0 \\
\hline 0 & 0 & I_{p_{3}}
\end{array}\right],
$$

where $S_{i}$ is a $\left[\left(p_{1}+p_{2}\right) \times\left(p_{1}+p_{2}\right)\right]$ matrix, such that $\hat{S}_{i}$ transforms $R_{i}$ as follows:

$$
\hat{S}_{i}^{-1} R_{i} \hat{S}_{i-1}=\tilde{R}_{i}=\left[\begin{array}{c|cc|c}
R_{i}^{00} & * & * & * \\
\hline 0 & R_{i}^{22} & 0 & * \\
0 & 0 & R_{i}^{11} & * \\
\hline 0 & 0 & 0 & R_{i}^{33}
\end{array}\right],
$$

which is

$$
S_{i}^{-1}\left[\begin{array}{cc}
R_{i}^{11} & R_{i}^{12} \\
0 & R_{i}^{22}
\end{array}\right] S_{i-1}=\left[\begin{array}{cc}
R_{i}^{22} & 0 \\
0 & R_{i}^{11}
\end{array}\right]
$$

The problem is to find the appropriate matrix $S_{i}$ which satisfies the above condition. Assume $S_{i}$ has form

$$
S_{i}=\left[\begin{array}{cc}
X_{i} & I_{p_{1}} \\
I_{p_{2}} & 0
\end{array}\right]
$$

where matrix $X_{i}$ has dimension $\left[p_{1} \times p_{2}\right]$. We obtain periodic Sylvester equation [17]

$$
R_{i}^{11} X_{i-1}-X_{i} R_{i}^{22}=-R_{i}^{12}, \quad i=0,1,2, \cdots, m .
$$

The algorithm to find eigenvectors is based on (5.3). If the $i_{t h}$ eigenvalue of $\mathbf{R}^{(k)}$ is real, we only need to exchange the first $[(i-1) \times(i-1)]$ block of $R_{k}, k=1,2, \cdots, m$ with its $i_{\text {th }}$ diagonal element. If the $i_{t h}$ and $(i+1)_{t h}$ eigenvalues form a complex pair, then the first $[(i-1) \times(i-1)]$ block and the following $[2 \times 2]$ block should be exchanged. Therefore $X_{i}$ in (5.3) has dimension $\left[p_{1} \times 1\right]$ or $\left[p_{1} \times 2\right]$. In both cases, $p_{0}=0$.

Real eigenvectors. In this case, matrix $X_{i}$ is just a column vector, so (5.3) is equivalent to

$$
\left[\begin{array}{ccccc}
R_{1}^{11} & -R_{1}^{22} I_{p_{1}} & & & \\
& R_{2}^{11} & -R_{2}^{22} I_{p_{1}} & & \\
& & R_{3}^{11} & -R_{3}^{22} I_{p_{1}} & \\
& & \ddots & \cdots \\
-R_{m}^{22} I_{p_{1}} & & & R_{m}^{11}
\end{array}\right]\left[\begin{array}{c}
X_{0} \\
X_{1} \\
X_{2} \\
\cdots \\
X_{m-1}
\end{array}\right]=\left[\begin{array}{c}
-R_{1}^{12} \\
-R_{2}^{12} \\
-R_{3}^{12} \\
\cdots \\
-R_{m}^{12}
\end{array}\right]
$$

where $R_{i}^{22}$ is the $\left(p_{1}+1\right)_{t h}$ diagonal element of $R_{i}$. The accuracy of eigenvectors is determined by the accuracy of solving sparse linear equation (5.4). In our application to periodic orbits in 
one dimensional Kuramoto-Sivashinsky equation, Gaussian elimination with partial pivoting (GEPP) is enough. For a more technical treatment, such as cyclic reduction or preconditioned conjugate gradients, to name a few, please see $[1,11,16]$.

Now we get all vectors $X_{i}$ by solving periodic Sylvester equation, but how are they related to the eigenvectors? In analogy to $\mathbf{R}^{(0)}$, defining $\tilde{\mathbf{R}}_{0}=\tilde{R}_{m} \tilde{R}_{m-1} \cdots \tilde{R}_{1}$, we get $\hat{S}_{m}^{-1} \mathbf{R}^{(0)} \hat{S}_{m}=$ $\tilde{\mathbf{R}}_{0}$ by (5.2). Since $p_{0}=0$ and $p_{2}=1$ in (5.2), the first eigenvector of $\tilde{\mathbf{R}}_{0}$, the one corresponding to eigenvalue $\Lambda_{p_{1}+1}$ is $\tilde{e}=(1,0, \cdots, 0)^{\top}$. Before normalization, the corresponding eigenvector of $\mathbf{R}^{(0)}$ is

$$
v_{p_{1}+1}^{(0)}=\hat{S}_{m} \tilde{e}=\left[X_{0}^{\top}, 1,0,0, \cdots, 0\right]^{\top} .
$$

This is the eigenvector of matrix $\mathbf{R}^{(0)}=R_{m} R_{m-1} \cdots R_{1}$ in (3.4) for $k=0$. For $\mathbf{R}^{(1)}=$ $R_{1} R_{m} \cdots R_{2}$, the corresponding periodic Sylvester equation will be cyclically rotated one row up, which means $X_{1}$ will be shifted to the first place in the column vector in (5.4), and thus the corresponding eigenvector of $\mathbf{R}^{(1)}$ is $v_{p_{1}+1}^{(1)}=\left[X_{1}^{\top}, 1,0, \cdots, 0\right]^{\top}$. The same argument goes for all the following $\mathbf{R}^{(k)}, k=2,3, \cdots, m-1$. In conclusion, solution of (5.4) contains the eigenvectors for all $\mathbf{R}^{(k)}, k=0,1, \cdots, m-1$. Another benefit of reordering method is that we can selectively get the eigenvectors corresponding to some specific eigenvalues. This merit is important in high dimensional nonlinear systems for which only a subset of Floquet vectors suffices to characterize the dynamics in tangent space, and thus we avoid wasting time in calculating the remaining transient uniformly vanishing modes.

Complex eigenvector pairs. As in the real eigenvalue case, we have $p_{0}=0$, but now $p_{2}=2$, so matrix $X_{i}$ has dimension $\left[p_{1} \times 2\right]$. Using the same notation as ref. [17], let $v\left(X_{i}\right)$ denote the vector representation of $X_{i}$ with the columns of $X_{i}$ stacked on top of each other, and let $A \otimes B$ denote the Kronecker product of two matrices, with the $(i, j)$-block element be $a_{i j} B$.

Now, the periodic Sylvester equation (5.3) is equivalent to

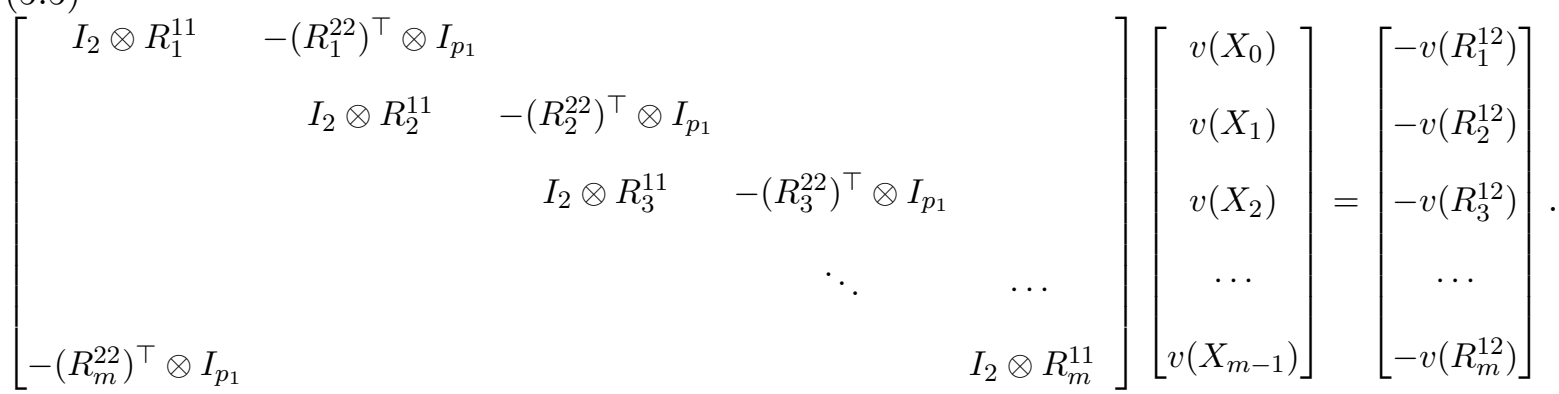

After switching $R_{i}^{11}$ and $R_{i}^{22}$, we can get the first two eigenvectors of $\tilde{\mathbf{R}}_{0}$ by multiplying the first $[2 \times 2]$ diagonal blocks of $\tilde{R}_{i}: R^{22}=R_{m}^{22} R_{m-1}^{22} \cdots R_{1}^{22}$. Let the eigenvectors of $R^{22}$ be $v$ and $v^{*}$ of size $[2 \times 1]$, then the corresponding eigenvectors of $\tilde{\mathbf{R}}_{0}$ are $\tilde{e}_{1}=\left(v^{\top}, 0,0, \cdots, 0\right)^{\top}$ and $\tilde{e}_{2}=\left(\tilde{e}_{1}\right)^{*}$ (the additional zeros make the length of the eigenvectors to be $n$ ). Therefore, the 
corresponding eigenvectors of $\mathbf{R}^{(0)}$ are

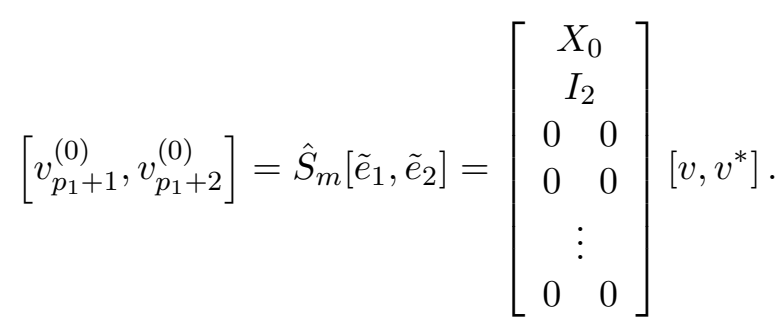

For other $\mathbf{R}^{(k)}$, the same argument in the real case applies here too, so we obtain all the complex eigenvector pairs for $\mathbf{R}^{(k)}, k=1,2, \cdots, m$.

6. Computational complexity and convergence analysis. In this paper we make no attempt at conducting a strict error analysis of the algorithms presented. However, for practical applications it is important to understand their computational costs. Periodic eigendecomposition is conducted in two stages: (1) periodic real Schur form, and (2) determination of all eigenvectors. In each stage, there are two candidate algorithms, so the efficiency of periodic eigendecomposition depends on the choice of the specific algorithm chosen in each stage.

Periodic QR algorithm and simultaneous iteration are both effective to achieve PRSF for the real eigenvalues, and for complex pairs of eigenvalues. Periodic QR algorithm consists of two stages. First, matrix sequence $J_{m}, J_{m-1} \cdots, J_{1}$ is reduced to Hessenberg-triangular form, with $J_{m-1}, \cdots, J_{1}$ upper triangular and $J_{m}$ upper Hessenberg. It requires $O(m n)$ Householder reflections in this stage and computational cost associated with each reflection is $O\left(n^{2}\right)$, if the transformed matrix is calculated implicitly without forming the Householder matrix [37]. So the overall computational cost of this stage is $O\left(m n^{3}\right)$. The second stage is the periodic QR iteration which is a generalization of the standard, $m=1$, case [37]. $O(m n)$ Givens rotations are performed in each iteration with overall computational cost of $O\left(m n^{2}\right)$. Though the computational effort in each iteration in the second stage is less than that in the first stage, the number of iterations in the second stage is usually far more than the dimension of matrices involved. In this sense, the second stage is the heavy part of periodic QR algorithm. On the other hand, simultaneous iteration conducts $m$ QR decomposition $O\left(m n^{3}\right)$ and $m$ matrixmatrix multiplication $O\left(m n^{3}\right)$ in each iteration, giving a total computational cost of $O\left(m n^{3}\right)$. The convergence of either algorithm depends linearly on the ratio of adjacent eigenvalues of $\mathbf{R}^{(0)}:\left|\Lambda_{i}\right| /\left|\Lambda_{i+1}\right|$ without shift [12]. Therefore the ratio of costs is approximately of the order $O\left(m n^{3}\right) / O\left(m n^{2}\right)=O(n)$, implying that the periodic QR algorithm is much cheaper than the simultaneous iteration if the dimension of matrices involved is large enough.

The second stage of periodic eigendecomposition is to find all the eigenvectors of $\mathbf{J}^{(k)}$ via quasi-upper triangular matrices $\mathbf{R}^{(k)}$. The first candidate is the combination of power iteration and shifted power iteration. The computational cost of one iteration for the $i_{\text {th }}$ eigenvector is $O\left(m i^{2}\right)$. The second candidate, reordering method, relies on an effective method to solve periodic Sylvester equation (5.3). For example, GEPP is suitable for well conditioned matrix (5.4) and (5.5) with computational cost of $O\left(m n^{2}\right)$. On the other hand, the iteration method, as pointed out earlier, could not produce the eigenvectors of $\mathbf{R}^{(k)}$ for all $k=1,2, \cdots, m$ accurately in the same time due to the noise introduced during the transformation process 
(3.4), especially when the magnitudes of eigenvalues span a large range. In contrast, the reordering algorithm is not iterative and it gives all the eigenvectors simultaneously.

In summary, if we just consider the computational effort, the combination of periodic QR algorithm and reordering method is preferable for periodic eigendecomposition.

7. Application to Kuramoto-Sivashinsky equation. Our ultimate goal of implementing periodic eigendecomposition is to analyze the stability of periodic orbits and the associated stable/unstable manifolds in dynamical systems, for the hope of getting a better understanding of pattern formation and turbulence. As an example, we focus on the one-dimensional Kuramoto-Sivashinsky equation

$$
u_{t}+\frac{1}{2}\left(u^{2}\right)_{x}+u_{x x}+u_{x x x x}=0, x \in[0, L]
$$

on a periodic spatial domain of size $L=22$, large enough to exhibit complex spatiotemporal dynamics. This equation is formulated independently by Kuramoto in the context of angular phase turbulence in reaction-diffusion systems [25], and by Sivashinsky in the study of hydrodynamic instability in laminar flames [28]. Periodic boundary condition enables us to transform this partial differential equation into a set of ODEs in Fourier space

$$
\dot{a}_{k}=\left(q_{k}^{2}-q_{k}^{4}\right) a_{k}-i \frac{q_{k}}{2} \sum_{m=-\infty}^{\infty} a_{m} a_{k-m}
$$

where $q_{k}=2 \pi k / L$, and the coefficients are complex, $a_{k}=b_{k}+i c_{k}$. In our simulations, discrete Fourier transform is used with $N=64$ modes $(k=-N / 2+1$ up to $N / 2$ in (7.2)).

Since $u(x, t)$ is real, $a_{k}(t)=a_{-k}^{*}(t)$; thus only half of the Fourier modes are independent. As $\dot{a}_{0}=0$ from (7.2), we can set $a_{0}=0$ corresponding to zero mean velocity without lose of generality. Also the nonlinear term of $\dot{a}_{N / 2}$ in fact has coefficient $q_{N / 2}+q_{-N / 2}=0$ from symmetric consideration [36]; thus $a_{N / 2}$ is decoupled from other modes and it can be set to zero as well. Thus then the number of independent variables is $N-2$,

$$
\hat{u}=\left(b_{1}, c_{1}, b_{2}, c_{2}, \cdots, b_{N / 2-1}, c_{N / 2-1}\right)^{\top} .
$$

This is the 'state space' in the discussion that follows. Exponential time-differencing scheme combined with RK4 $[6,21]$ is implemented to integrate $(7.2)$. The combination of periodic QR algorithm algorithm and reordering algorithm is used to obtain all exponents and eigenvectors. In addition, Gaussian elimination with partial pivoting (GEPP) is stable for (5.4) and (5.5) if the time step in Kuramoto-Sivashinsky integrator is not too large, as GEPP only uses addition and subtraction operations.

Kuramoto-Sivashinsky equation is equivariant under reflection and space translation: $-u(-x, t)$ and $u(x+l, t)$ are also solutions if $u(x, t)$ is a solution, which corresponds to equivariance of (7.3) under group operation $R=\operatorname{diag}(-1,1,-1,1, \cdots)$ and $g(l)=\operatorname{diag}\left(r_{1}, r_{2}, \cdots, r_{N / 2-1}\right)$, where

$$
r_{k}=\left(\begin{array}{cc}
\cos \left(q_{k} l\right) & -\sin \left(q_{k} l\right) \\
\sin \left(q_{k} l\right) & \cos \left(q_{k} l\right)
\end{array}\right), \quad k=1,2, \cdots, N / 2-1 .
$$

Based on the consideration of these symmetries, there are three types of invariant orbits in Kuramoto-Sivashinsky system: periodic orbits in the $b_{k}=0$ invariant antisymmetric subspace, 

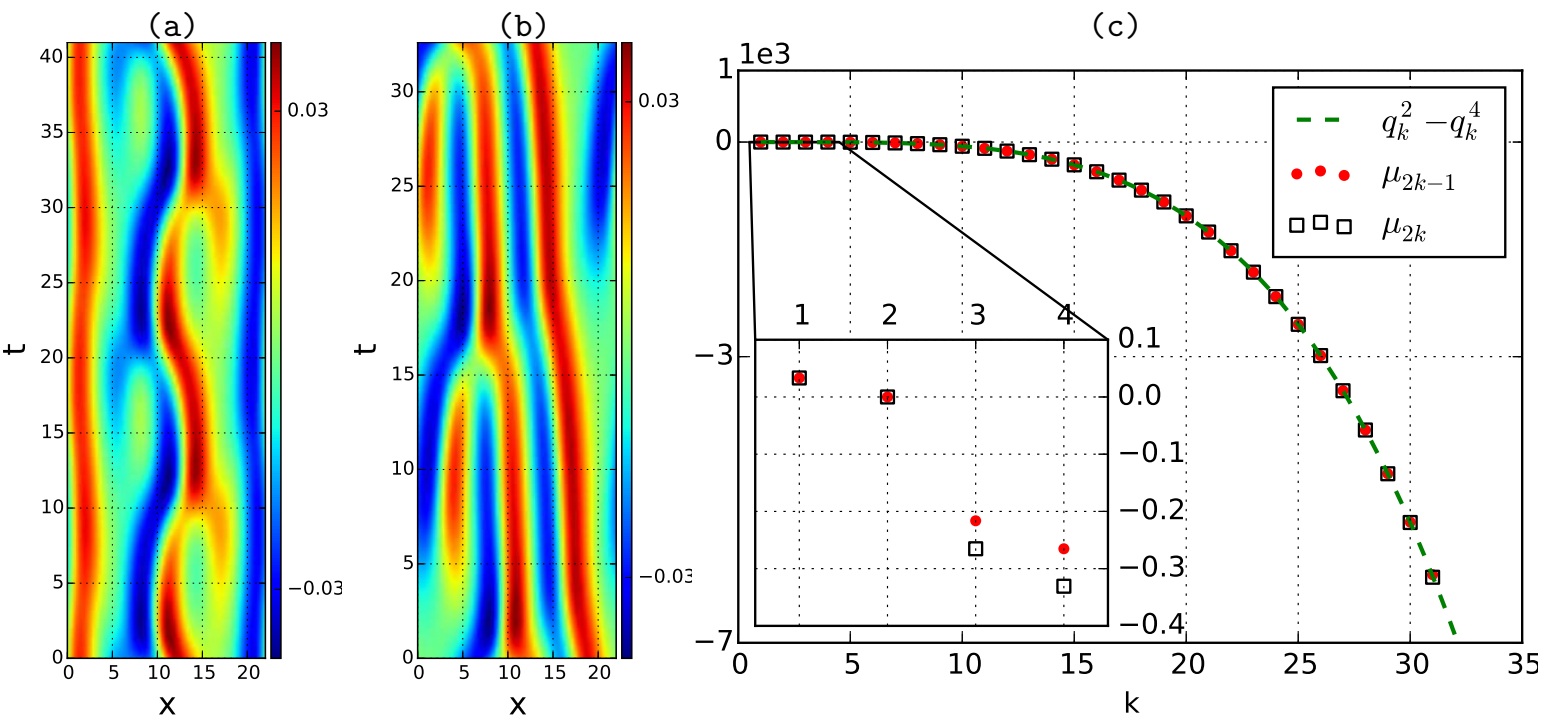

Figure 3. (Color online) (a) Preperiodic orbit $\overline{p p}_{10.25}$ and (b) relative periodic orbit $\overline{r p}_{16.31}$ for total evolution time $4 T_{p p}$ and $2 T_{r p}$, respectively. The phase shift for $\overline{r p}_{16.31}$ after one prime period $\simeq-2.863$. (c) The real parts of Floquet exponents paired for a given $k$ as $\left(k, \mu_{2 k-1}\right)$ and $\left(k, \mu_{2 k}\right)$, for $\overline{p p}_{10.25}$ with truncation number $N=64$. The dashed line (green) is $q_{k}^{2}-q_{k}^{4}$. The inset is a magnification of the region containing the 8 leading entangled modes.

preperiodic orbits which are self-dual under reflection, and relative periodic orbits with a shift along group orbit after one period. As shown in ref. [8], the first type is absent for domains as small as $L=22$, and thus we focus on the last two types of orbits. For preperiodic orbits $\hat{u}(0)=R \hat{u}\left(T_{p}\right)$, we only need to evolve the system for a prime period $T_{p}$ which is half of the whole period, with the Floquet matrix given by $J_{p}(\hat{u})=R J^{T_{p}}(\hat{u})$. A relative periodic orbit, $\hat{u}(0)=g_{p} \hat{u}\left(T_{p}\right)$, returns after one period $T_{p}$ to the initial state upon the group transform $g_{p}=g\left(l_{p}\right)$, so the corresponding Floquet matrix is $J_{p}(\hat{u})=g_{p} J^{T_{p}}(\hat{u})$. Here we show how periodic eigendecomposition works by applying it to one representative preperiodic orbit $\overline{p p}_{10.25}$ and two relative periodic orbits $\overline{r p}_{16.31}$ and $\overline{r p}_{57.60}$ (subscript indicates the period of the orbit), described in ref. [8].

Figure 3 shows the time evolution of $\overline{p p}_{10.25}$ and $\overline{r p}_{16.31}$ and the Floquet spectrum of $\overline{p p}_{10.25}$. At each repeat of the prime period, $\overline{p p}_{10.25}$ is invariant under reflection along $x=L / 2$, figure 3 (a), and $\overline{r p}_{16.31}$ has a shift along the $x$ direction as time goes on, figure $3(\mathrm{~b})$. Since $\overline{p p}_{10.25}$ and $\overline{r p}_{16.31}$ are both time invariant and equivariant under $\mathrm{SO}(2)$ group transformation $g(l)$, there should be two marginal Floquet exponents, corresponding to the velocity field $v(x)$ and group tangent $t(x)=\mathbf{T} x$ respectively, where $\mathbf{T}$ is the generator of $\mathrm{SO}(2)$ rotation:

$$
\mathbf{T}=\operatorname{diag}\left(t_{1}, t_{2}, \cdots, t_{N / 2-1}\right), \quad t_{k}=\left(\begin{array}{cc}
0 & -q_{k} \\
q_{k} & 0
\end{array}\right) .
$$

Table 1 shows that the $2_{n d}$ and $3_{r d}$, respectively $3_{r d}$ and $4_{t h}$ exponents of $\overline{r p}_{16.31}$, respectively $\overline{p p}_{10.25}$, are marginal, with accuracy as low as $10^{-12}$, to which the inaccuracy introduced by the error in the closure of the orbit itself also contributes. Table 1 and figure 3 (c) show that periodic Schur decomposition is capable of resolving Floquet multipliers differing by thousands 
Table 1

The first 10 and last four Floquet exponents and Floquet multiplier phases, $\Lambda_{i}=\exp \left(T \mu_{i} \pm i \theta_{i}\right)$, for orbits $\overline{p p}_{10.25}$ and $\overline{r p}_{16.31}$, respectively. $\theta_{i}$ column lists either the phase, if the Floquet multiplier is complex, or '-1' if the multiplier is real, but inverse hyperbolic. Truncation number $N=64$. The 8 leading exponents correspond to the entangled modes: note the sharp drop in the value of the $9_{\text {th }}$ and subsequent exponents, corresponding to the isolated modes.

\begin{tabular}{llc|llc} 
& \multicolumn{1}{c|}{$\overline{p p}_{10.25}$} & \multicolumn{3}{|c}{$\overline{r p}_{16.31}$} \\
$i$ & \multicolumn{1}{c}{$\mu_{i}$} & $\theta_{i}$ & $i$ & \multicolumn{1}{c}{$\mu_{i}$} & $\theta_{i}$ \\
\hline 1,2 & 0.033209 & \pm 2.0079 & 1 & 0.32791 & \\
3 & $-4.1096 \mathrm{e}-13$ & & 2 & $2.8679 \mathrm{e}-12$ & \\
4 & $-3.3524 \mathrm{e}-14$ & -1 & 3 & $2.3559 \mathrm{e}-13$ & \\
5 & -0.21637 & & 4 & -0.13214 & -1 \\
6,7 & -0.26524 & \pm 2.6205 & 5,6 & -0.28597 & \pm 2.7724 \\
8 & -0.33073 & -1 & 7 & -0.32821 & -1 \\
9 & -1.9605 & & 8 & -0.36241 & \\
10 & -1.9676 & -1 & 9,10 & -1.9617 & \pm 2.2411 \\
$\ldots$ & $\ldots$ & $\cdots$ & $\cdots$ & $\cdots$ & $\cdots$ \\
59 & -5313.6 & -1 & 59 & -5314.4 & \\
60 & -5317.6 & & 60 & -5317.7 & \\
61 & -6051.8 & -1 & 61 & -6059.2 & \\
62 & -6080.4 & & 62 & -6072.9 & \\
\hline
\end{tabular}

of orders: when $N=64$, the smallest Floquet multiplier for $\overline{p p}_{10.25}$ is $\left|\Lambda_{62}\right| \simeq e^{-6080.4 \times 10.25}$. We should know that this cannot be achieved if we try to get a single Jacobian for the whole orbit. Figure 3(c) and table 1 also show that for $k \geq 9$, Floquet exponents almost lie on the curve $\left(q_{k}^{2}-q_{k}^{4}\right)$. This is the consequence of strong dissipation caused by the linear term in (7.2) for large Fourier mode index. This feature is observed for all the other periodic orbits we have experimented, and it is a good indicator of existence of a finite dimensional inertial manifold. Also, Floquet exponents appear in pairs for large indices simply because the real and complex part of high Fourier modes have similar contracting rate from (7.2).

(a)

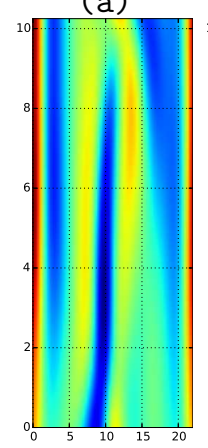

(b)

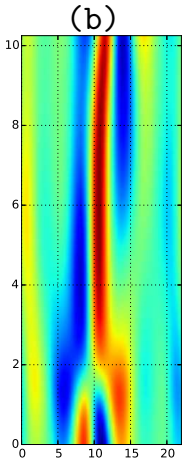

(c)

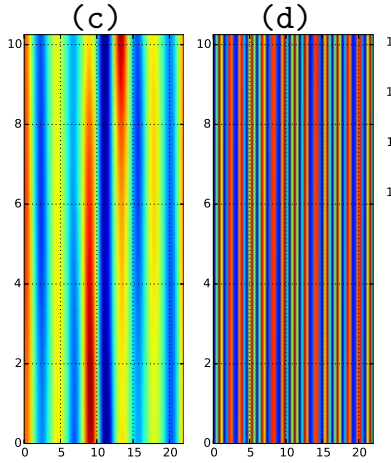

(e)

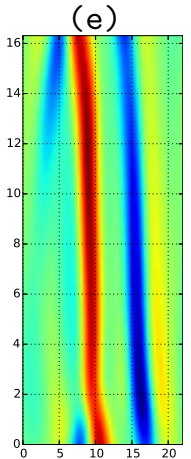

(f)

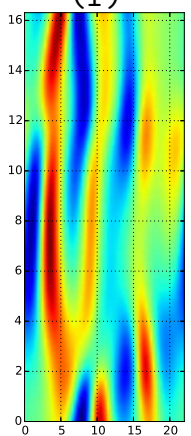

(g)

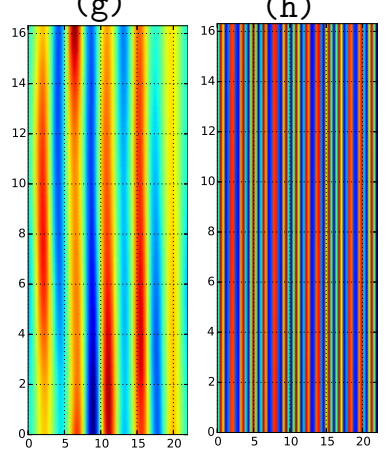

Figure 4. (Color online) $(a) \sim(d)$ : the 1st (real part), 5th, 10 th and 30th Floquet vector along $\overline{p p}_{10.25}$ for one prime period. $(e) \sim(h)$ : the 1st, 4 th (real part), 10th (imaginary part) 30th (imaginary part) Floquet vector along $\overline{r p}_{16.31}$ for one prime period. axes and color scale are the same with figure 3.

Figure 4 shows a few selected Floquet vectors along $\overline{p p}_{10.25}$ and $\overline{r p}_{16.31}$ for one prime period respectively. We need to remind the reader that Floquet vectors for a whole period 

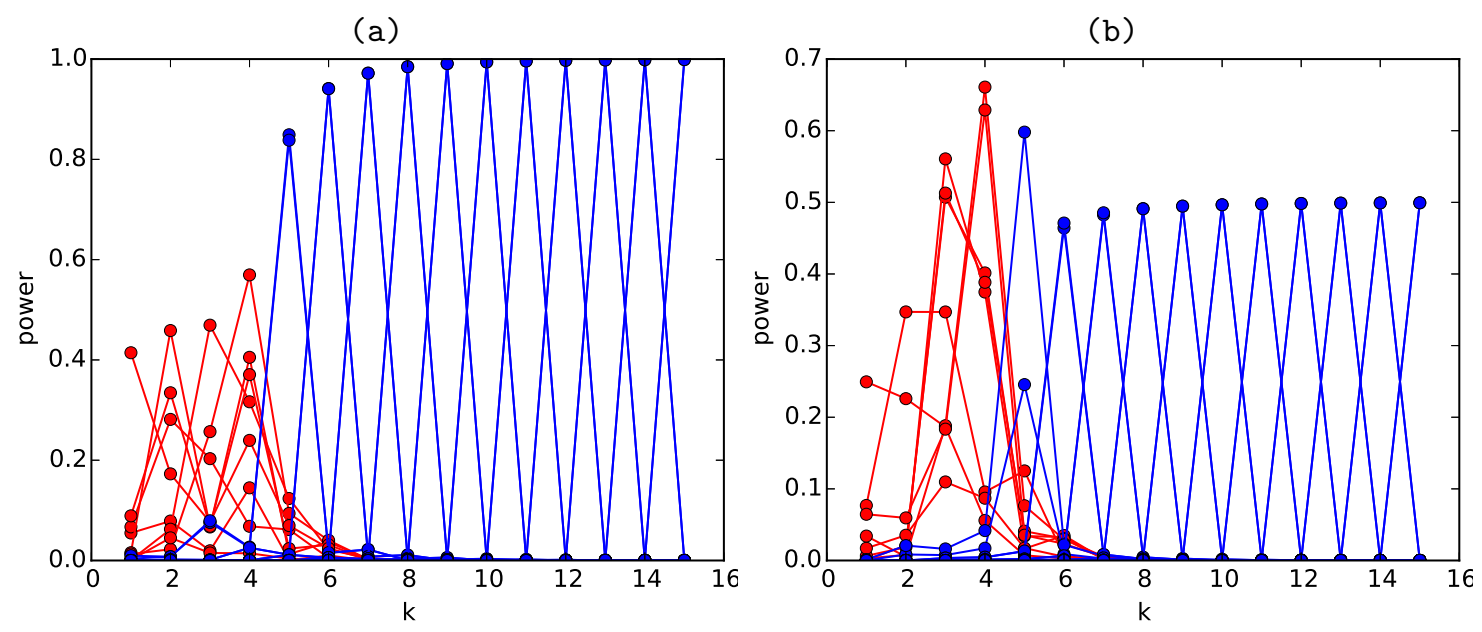

Figure 5. (Color online) The power spectrum of the first 30 Floquet vectors for $\overline{p p}_{10.25}$ (left) and $\overline{r p}_{16.31}$ (right) at $t=0$. Red lines corresponds to the leading 8 Floquet vectors; while the blue lines correspond to the left 22 Floquet vectors with the ith one localized at index $i$. Power is defined to be the modular square of Fourier coefficients of Floquet vectors. The $x$-axis is labeled by the Fourier mode indices. Only the $k>0$ part is shown, the negative $k$ follow by reflection. For complex Floquet vectors, the power spectra of real part and imaginary part are calculated separately. Since almost all contracting Floquet vectors of $\overline{r p}_{16.31}$ form complex conjugate pairs, their power peaks are far less than 1.

is obtained by solving (5.4) or (5.5), not by evolving Floquet vectors at one time spot to the later time spots because the evolution procedure is not stable for Floquet vectors. We can see that the leading few Floquet vectors have turbulent structures containing only long waves for both $\overline{p p}_{10.25}$ and $\overline{r p}_{16.31}$, but for Floquet vectors corresponding to strong contracting rates, the configurations are pure sinusoidal curves. The power spectra in Figure 5 demonstrate this point too. The leading 8 Floquet vectors have large components in the first 5 Fourier modes and the spectra are entangled with each other; while the remaining Floquet vectors almost concentrate on a single Fourier mode and are decoupled from each other; more specifically, the $i$ th Floquet vector with $i \geq 9$ peaks at the $\left\lceil\frac{i}{2}\right\rceil$ th mode in figure 5 . Takeuchi etc. [34, 42] observe similar features in covariant vectors along ergodic trajectories and by measuring the tangency between these two groups of covariant vectors, they reach a reasonable conclusion about the dimension of inertial manifold of Kuramoto-Sivashinsky equation and complex GinzburgLandau equation. Therefore, we anticipate that by analyzing the tangency of Floquet vectors along different periodic orbits can also lead to the same conclusion, which is our further research.

We have noted above that the group property of Jacobian matrix multiplication (2.1) enables us to factorize $\mathbf{J}^{(k)}$ into a product of short-time matrices with matrix elements of comparable magnitudes. In practice, caution should be exercised when trying to determine the optimal number of time increments that the orbit should be divided into. If the number of time increments $m$ is too large, then, according to the estimates of sect. 6 , the computation may be too costly. If $m$ is too small, then the elements of Jacobian matrix corresponding to the corresponding time increment may range over too many orders of magnitude, causing periodic eigendecomposition to fail to resolve the most contracting Floquet vector along the 

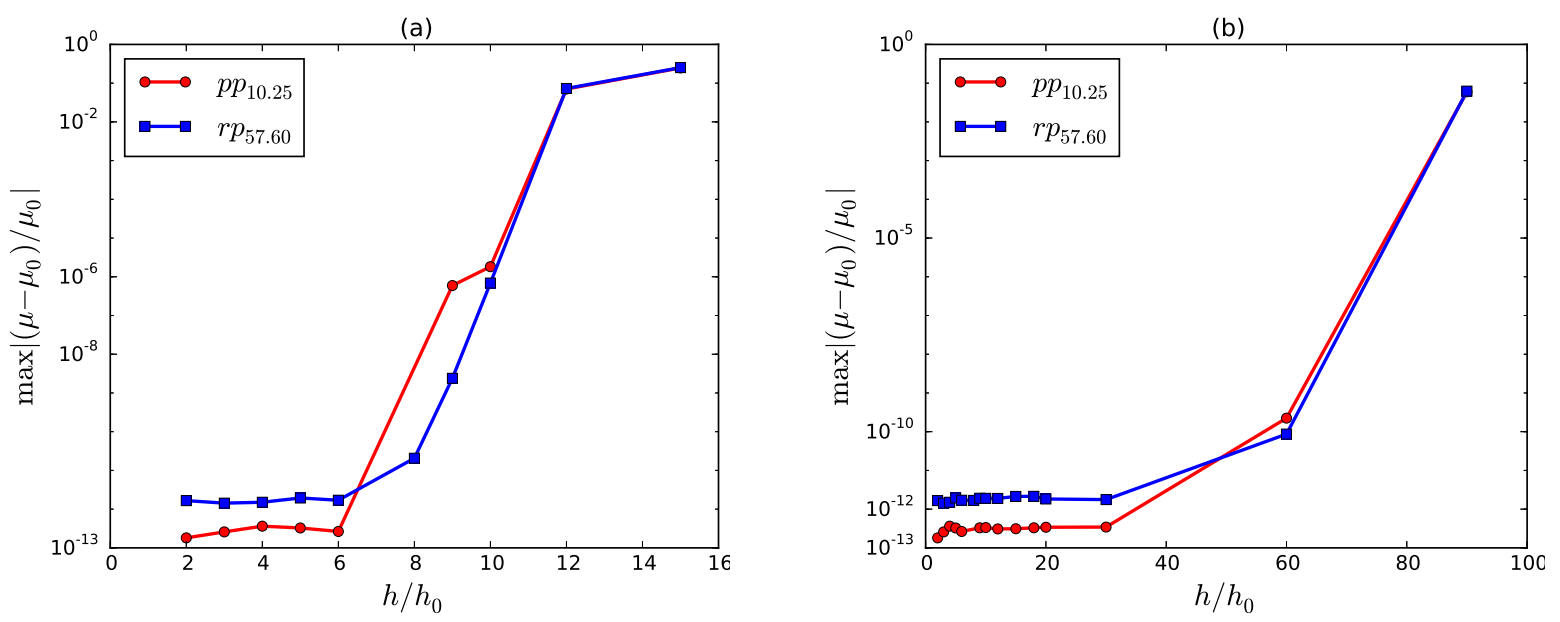

Figure 6. (Color online) Relative error of the real part of Floquet exponents associated with different time steps with which the Floquet matrix is integrated. Two orbits $\overline{p p}_{10.25}$ and $\overline{r p}_{57.60}$ are used as an example with the base case $h_{0} \approx 0.001$. (a) The maximal relative difference of the whole set of Floquet exponents with increasing time step (decreasing the number of ingredient segments of the orbit). (b) Only consider the first 35 Floquet exponents.

orbit. One might also vary the time step according to the velocity at a give point on the orbit. Here we determined satisfactory $m$ 's by numerical experimentation shown in figure 6 . Since larger time step means fewer time increments of the orbit, a very small time step $\left(h_{0} \approx 0.001\right)$ is chosen as the base case, and it is increased to test whether the corresponding Floquet exponents change substantially or not. As shown in figure 6 (a), up to $6 h_{0}$ the whole Floquet spectrum varies within $10^{-12}$ for both $\overline{p p}_{10.25}$ and $\overline{r p}_{57.60}$. These two orbits represent two different types of invariant solutions which have short and long periods, so we presume that time step $6 h_{0}$ is good enough for other short or long orbits too. On the other hand, if only the first few Floquet exponents are desired, the time step can be increased further to fulfill the job. As shown in figure 6 (b), if we are only interested in the first 35 Floquet exponents, then time step $30 h_{0}$ is small enough. In high dimensional nonlinear systems, often we are not interested in the dynamics in the very contracting directions because they are usually decoupled from the physical modes, and shed little insight into the system properties. Therefore, large time step could to used in order to save time.

The two marginal directions have a simple geometrical interpretation and provides a metric for us to measure the convergence of periodic eigendecomposition. Figure 7 (a) depicts the two marginal vectors of $\overline{p p}_{10.25}$ projected onto the subspace spanned by $\left[a_{1}, b_{1}, a_{2}\right]$ (the real, imaginary parts of the first mode and the real part of the second Fourier mode). The first marginal eigen-direction (the $3_{r d}$ Floquet vector in table 1 ) is aligned with the velocity field along the orbit, and the second marginal direction (the $4_{t h}$ Floquet vector) is aligned with the group tangent. The numerical difference between the unit vectors along these two marginal directions and the corresponding physical directions is shown in figure 7 (b). The difference is under $10^{-9}$ and $10^{-11}$ for these two directions, which demonstrates the accuracy of the algorithm. As shown in table 1 , for an preperiodic orbit, such as $\overline{p p}_{10.25}$, the trajectory 
(a)

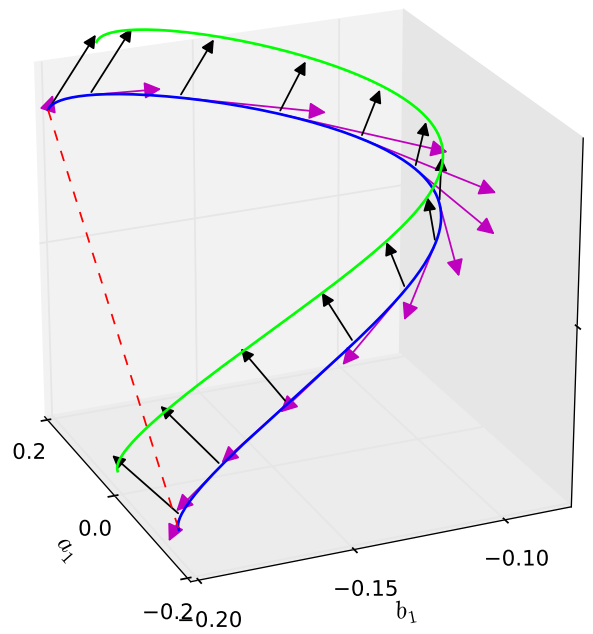

(b)

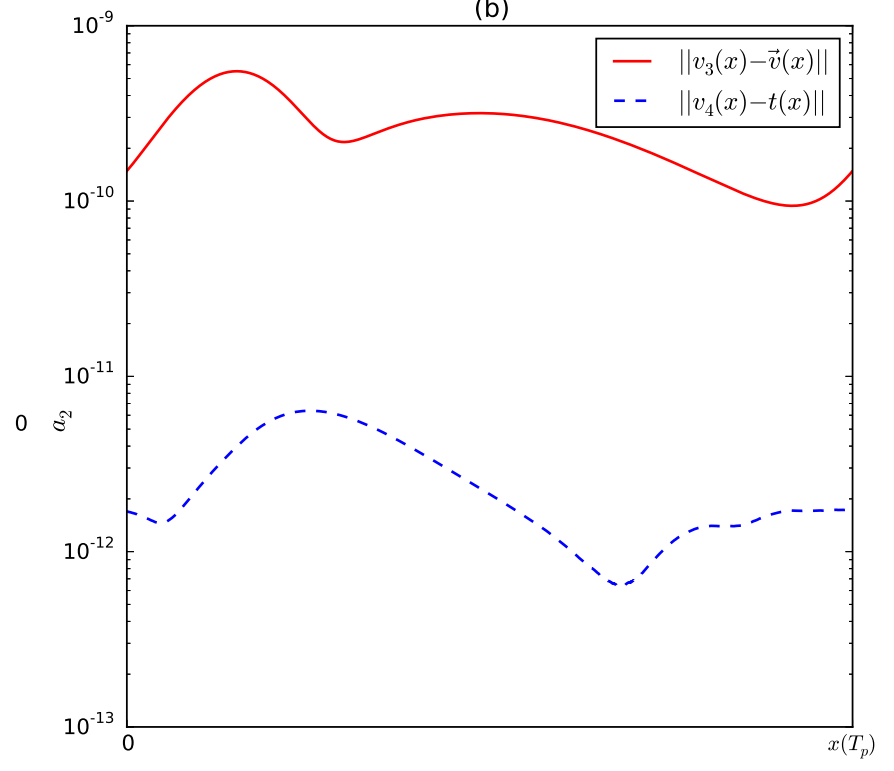

Figure 7. (Color online) Marginal vectors and the associated errors. (a) $\overline{p p}_{10.25}$ in one period projected onto $\left[a_{1}, b_{1}, a_{2}\right]$ subspace (blue curve), and its counterpart (green line) generated by a small group transformation $g(\ell)$, here arbitrarily set to $\ell=L /(20 \pi)$. Magenta and black arrows represent the first and the second marginal Floquet vectors $\mathbf{e}_{3}(x)$ and $\mathbf{e}_{4}(x)$ along the prime orbit. (b) The solid red curve is the magnitude of the difference between $\mathbf{e}_{3}(x)$ and the velocity field $\vec{v}(x)$ along the orbit, and blue dashed curve is the difference between $\mathbf{e}_{4}(x)$ and the group tangent $t(x)=\mathbf{T} x$.

tangent and the group tangent have eigenvalue +1 and -1 respectively, and are thus distinct. However, the two marginal directions are degenerate for an relative periodic orbit, such as $\overline{r p}_{16.31}$. So these two directions are not fixed, but the plane that they span is uniquely determined. Figure 8 shows the velocity field and group tangent along orbit $\overline{r p}_{16.31}$ indeed lie in the subspace spanned by these two marginal directions.

8. Conclusion and future work. In this paper, as well as in the forthcoming publication, ref. [9], we use one-dimensional Kuramoto-Sivashinsky system to illustrate the effectiveness and potential wide usage of periodic eigendecomposition applied to stability analysis in dissipative nonlinear systems.

On the longer time scale, we hope to apply the method to the study of orbits of much longer periods, as well as to the study of high-dimensional, numerically exact time-recurrent unstable solutions of the full Navier-Stokes equations. Currently up to 30 Floquet vectors for plane Couette invariant solutions can be computed [13], but many more will be needed and to a higher accuracy in order to determine the physical dimension of a turbulent Navier-Stokes flow. We are nowhere there yet; we anticipate the need for optimizing and parallelizing such algorithms. Also there is opportunity to apply periodic eigendecomposition to Hamiltonian systems too and we need additional tests to show its ability to preserve symmetries of Floquet spectrum imposed by Hamiltonian systems. 


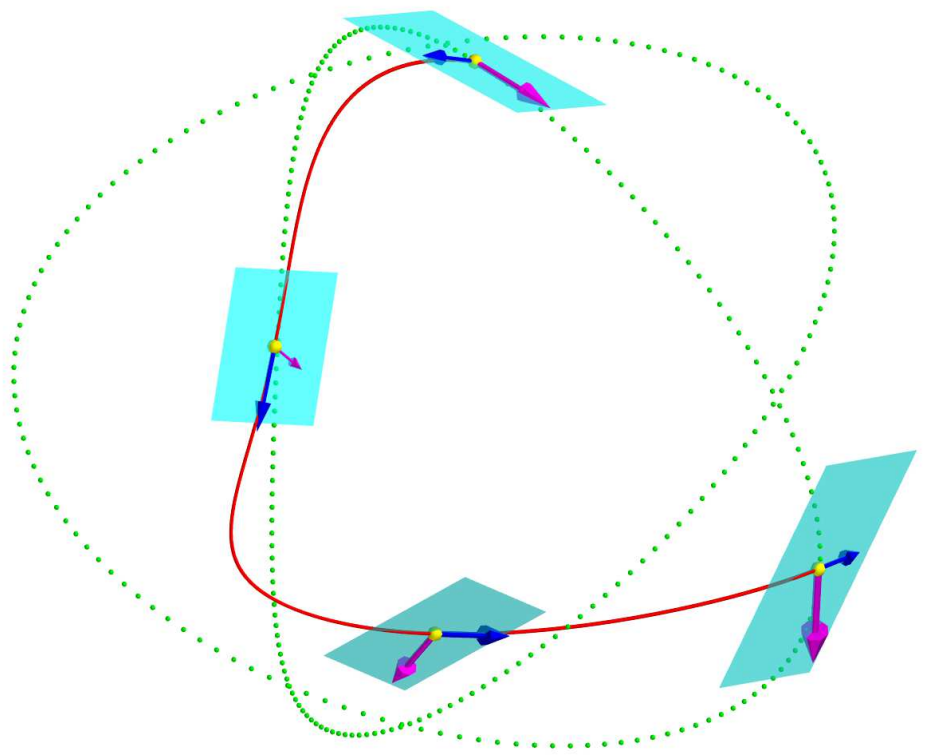

Figure 8. (Color online) Projection of relative periodic orbit $\overline{r p}_{16.31}$ onto the Fourier modes subspace $\left[b_{2}, c_{2}, b_{3}\right]$ (red curve). The dotted curve (lime) is the group orbit connecting the initial and final points. Blue and magenta arrows represent the velocity field and group tangent along the orbit, respectively. Two-dimensional planes (cyan) are spanned by the two marginal Floquet vectors at each point (yellow) along the orbit.

Acknowledgments. We are grateful to L. Dieci for introducting us to complex periodic Schur decomposition, K.A. Takeuchi for providing detailed documentation of his previous work on covariant vectors, R.L. Davidchack for his database of periodic orbits in KuramotoSivashinsky equation, which are the basis of our numerical experiments, and to N.B. Budanur, E. Siminos, M.M. Farazmand and H. Chaté for many spirited exchanges, X.D. was supported by NSF grant DMS-1028133. P.C. thanks G. Robinson, Jr. for support.

\section{REFERENCES}

[1] P. Amodio, J. R. Cash, G. Roussos, R. W. Wright, G. Fairweather, I. Gladwell, G. L. Kraut, AND M. PAPRZYCKI, Almost block diagonal linear systems: sequential and parallel solution techniques, and applications, Numer. Linear Algebra Appl., 7 (2000), pp. 275-317.

[2] G. Benettin, L. Galgani, A. Giorgilli, and J. M. Strelcyn, Lyapunov characteristic exponents for smooth dynamical systems; a method for computing all of them. Part 1: theory, Meccanica, 15 (1980), pp. 9-20.

[3] A. Bojanczyk, G. H. Golub, And P. V. Dooren, The periodic Schur decomposition. Algorithms and applications, in Proc. SPIE Conference, vol. 1770, 1992, pp. 31-42.

[4] H. Bosetti, H. A. Posch, C. Dellago, And W. G. Hoover, Time-reversal symmetry and covariant lyapunov vectors for simple particle models in and out of thermal equilibrium, Phys. Rev. E, 82 (2010), pp. 1-10.

[5] F. Christiansen, P. Cvitanović, and V. Putkaradze, Spatiotemporal chaos in terms of unstable recurrent patterns, Nonlinearity, 10 (1997), pp. 55-70. arXiv: chao-dyn/9606016.

[6] S. M. Cox And P. C. Matthews, Exponential time differencing for stiff systems, J. Comput. Phys., 176 (2002), pp. 430-455. 
[7] P. Cvitanović, R. Artuso, R. Mainieri, G. Tanner, and G. Vattay, Chaos: Classical and Quantum, Niels Bohr Inst., Copenhagen, 2014. ChaosBook.org.

[8] P. Cvitanović, R. L. Davidchack, and E. Siminos, On the state space geometry of the Kuramoto-Sivashinsky flow in a periodic domain, SIAM J. Appl. Dyn. Syst., 9 (2010), pp. 1-33. arXiv:0709.2944.

[9] X. Ding, P. Cvitanović, K. A. Takeuchi, H. Chaté, E. Siminos, and R. L. Davidchack, Determination of the physical dimension of a dissipative system by periodic orbits. In preparation, 2015.

[10] S. V. Ershov And A. B. Potapov, On the concept of stationary Lyapunov basis, Physica D, 118 (1998), pp. $167-198$.

[11] G. Fairweather and I. Gladwell, Algorithms for almost block diagonal linear systems, SIAM Rev., 46 (2004), pp. 49-58.

[12] J. G. F. Francis, The QR transformation: A unitary analogue to the LR transformation. I, Comput. J., 4 (1961), pp. 265-271.

[13] J. F. Gibson, J. Halcrow, And P. Cvitanović, Visualizing the geometry of state-space in plane Couette flow, J. Fluid Mech., 611 (2008), pp. 107-130. arXiv:0705.3957.

[14] F. Ginelli, H. Chaté, R. Livi, And A. Politi, Covariant Lyapunov vectors, J. Phys. A, 46 (2013), p. 254005. arXiv:1212.3961.

[15] F. Ginelli, P. Poggi, A. Turchi, H. Chaté, R. Livi, And A. Politi, Characterizing dynamics with covariant Lyapunov vectors, Phys. Rev. Lett., 99 (2007), p. 130601. arXiv:0706.0510.

[16] R. Granat, I. Jonsson, And B. KÅgströM, Recursive blocked algorithms for solving periodic triangular Sylvester-type matrix equations, in Proc. 8th Intern. Conf. Applied Parallel Computing: State of the Art in Scientific Computing, PARA'06, 2007, pp. 531-539.

[17] R. GRanat And B. KÅgstRÖM, Direct eigenvalue reordering in a product of matrices in periodic Schur form, SIAM J. Matrix Anal. Appl., 28 (2006), pp. 285-300.

[18] J. Guckenheimer And P. Holmes, Nonlinear Oscillations, Dynamical Systems, and Bifurcations of Vector Fields, Springer, New York, 1983.

[19] M. Inubushi, M. U. Kobayashi, S.-I. TAkehiro, And M. Yamada, Covariant Lyapunov analysis of chaotic Kolmogorov flows, Phys. Rev. E, 85 (2012), p. 016331.

[20] M. Jolly, R. Rosa, and R. Temam, Evaluating the dimension of an inertial manifold for the KuramotoSivashinsky equation, Advances in Differential Equations, 5 (2000), pp. 31-66.

[21] A.-K. Kassam and L. N. Trefethen, Fourth-order time stepping for stiff PDEs, SIAM J. Sci. Comput., 26 (2005), pp. 1214-1233.

[22] M. KRUPA, Bifurcations of relative equilibria, SIAM J. Math. Anal., 21 (1990), pp. 1453-1486.

[23] P. V. Kuptsov, Violation of hyperbolicity via unstable dimension variability in a chain with local hyperbolic chaotic attractors, J. Phys. A, 46 (2013), p. 254016.

[24] P. V. Kuptsov and U. Parlitz, Theory and computation of Covariant Lyapunov Vectors, J. Nonlin. Sci., 22 (2012), pp. 727-762. arXiv:1105.5228.

[25] Y. Kuramoto And T. Tsuzuki, On the formation of dissipative structures in reactiondiffusion systems, Prog. Theor. Phys., 54 (1975), p. 687699.

[26] K. Lust, Improved numerical Floquet multipliers, Internat. J. Bifur. Chaos Appl. Sci. Engrg., 11 (2001), pp. 2389-2410.

[27] A. Lyapunov, Problème général de la stabilité du mouvement, Ann. of Math. Studies, 17 (1977). Russian original Kharkow, 1892

[28] D. M. Michelson AND G. I. Sivashinsky, Nonlinear analysis of hydrodynamic instability in laminar flames - II. numerical experiments, Acta Astronaut., 4 (1977), pp. 1207-1221.

[29] V. I. Oseledec, A multiplicative ergodic theorem. Liapunov characteristic numbers for dynamical systems, Trans. Moscow Math. Soc., 19 (1968), pp. 197-221.

[30] A. Politi, F. Ginelli, S. Yanchuk, And Y. Maistrenko, From synchronization to Lyapunov exponents and back, Physica D, 224 (2006), p. 90. arXiv:nlin/0605012.

[31] J. C. Robinson, Inertial manifolds for the Kuramoto-Sivashinsky equation, Phys. Lett. A, 184 (1994), pp. 190-193.

[32] D. RuELLe, Ergodic theory of differentiable dynamical systems, Publ. Math. IHES, 50 (1979), pp. 27-58.

[33] K. A. TAkeuchi, F. Gineldi, And H. Chaté, Lyapunov analysis captures the collective dynamics of large chaotic systems, Phys. Rev. Lett., 103 (2009), p. 154103. arXiv:0907.4298. 
[34] K. A. Takeuchi, H. Yang, F. Ginelli, G. Radons, and H. Chaté, Hyperbolic decoupling of tangent space and effective dimension of dissipative systems, Phys. Rev. E, 84 (2011), p. 046214. arXiv:1107.2567.

[35] R. Temam, Infinite-Dimensional Dynamical Systems in Mechanics and Physics, Springer, New York, 1988.

[36] L. N. Trefethen, Spectral Methods in MATLAB, SIAM, Philadelphia, 2000.

[37] L. N. Trefethen and D. Bau, Numerical Linear Algebra, SIAM, Philadelphia, 1997.

[38] D. S. Watkins, Francis's algorithm, Amer. Math. Monthly, 118 (2011), pp. 387-403.

[39] C. L. Wolfe And R. M. Samelson, An efficient method for recovering Lyapunov vectors from singular vectors, Tellus A, 59 (2007), pp. 355-366.

[40] H. Yang And G. Radons, Geometry of inertial manifold probed via Lyapunov projection method, Phys. Rev. Lett., 108 (2012), p. 154101.

[41] H.-L. YAng AND G. Radons, Comparison between covariant and orthogonal Lyapunov vectors, Phys. Rev. E, 82 (2010), p. 046204. arXiv:1008.1941.

[42] H.-L. Yang, K. A. Takeuchi, F. Ginelli, H. Chaté, and G. Radons, Hyperbolicity and the effective dimension of spatially-extended dissipative systems, Phys. Rev. Lett., 102 (2009), p. 074102. arXiv:0807.5073. 